\title{
Resolving the Partonic Structure of Nuclei through Energy-Frontier eA Scattering
}

\author{
Néstor Armesto* for the LHeC/FCC-eh Study Group ${ }^{\dagger}$ \\ Instituto Galego de Física de Altas Enerxías IGFAE, \\ Universidade de Santiago de Compostela, 15782 Santiago de Compostela, Galicia-Spain \\ E-mail: nestor.armesto@usc.es
}

The Large Hadron-electron Collider ( $\mathrm{LHeC}$ ) is a proposed upgrade of the Large Hadron Collider (LHC). An energy recovery linac would provide $60 \mathrm{GeV}$ electrons to collide with the proton and nuclear beams from the High Luminosity LHC (HL-LHC) and, eventually, with those from the High Energy LHC (HE-LHC) and the Future Circular Collider in hadron-hadron mode (FCChh). Working concurrently with the $\mathrm{pPb}$ or $\mathrm{PbPb}$ LHC modes, it will provide $\mathrm{ePb}$ collisions in the $\mathrm{TeV}$ regime with luminosities $\sim 10^{33} \mathrm{~cm}^{-2} \mathrm{~s}^{-1}$ to achieve integrated luminosities $\sim 10 \mathrm{fb}^{-1}$ in ten years. It will offer a huge extension of the kinematical coverage with respect to the one presently covered by data used in nuclear parton density functions (nPDF) fits, down to $x \sim 10^{-6}$ in the perturbative region for the LHeC. In this contribution I will show the possibilities in such machine for determining the nPDFs with unprecedented precision, in a region of interest for both HL-LHC and future AA colliders, with the possibility of extracting the complete set of PDFs of a single nucleus in a single experiment. Confronting such precision nPDFs with data from nuclear colliders will then set the eventual factorisation breaking effects and allow a more precise characterisation of the properties of the Quantum Chromodynamics (QCD) matter produced in such collisions. I will also comment on the possibilities for the extraction of diffractive parton densities in proton and nucleus.

International Conference on Hard and Electromagnetic Probes of High-Energy Nuclear Collisions 30 September - 5 October 2018

Aix-Les-Bains, Savoie, France

\footnotetext{
*Speaker.

${ }^{\dagger}$ http://lhec.web.cern.ch/, https://fcc.web.cern.ch/.
} 


\section{Introduction}

The distribution of partons inside nuclei, nuclear parton densities nPDFs, are essential ingredients of our understanding of nuclear structure at partonic level, see [1, 2]. For inclusive production, nPDFs determine particle production in collisions involving nuclei within the collinear factorisation framework. Therefore, their knowledge is required for the extraction of the properties of the dense hot partonic medium created in heavy-ion collisions, the Quark Gluon Plasma, through the comparison of particle yields and spectra in nucleus-nucleus and proton-proton collisions.

nPDFs suffer from large uncertainties [2], mainly due to the scarcity of data which cover a rather limited region of the $x-Q^{2}$ plane, see Fig. 1. Besides, the number of data for a single nucleus, e.g. $\mathrm{Pb}$, is very small and parametrisations interpolating between different nuclei are used in global fits $[3,4]$ that include Deep Inelastic Scattering (DIS) and Drell-Yan (DY) fixed target data, with electron, muon and neutrino beams, plus data from dAu collision at the Relativistic Heavy Ion Collider (RHIC) and pPb collisions at the LHC - less than 2000 data points for 14 nuclei to be contrasted with more than 4000 data points used in current global fits for proton PDFs. Several possibilities exist for improving this situation $[2,5,6]$ in future $\mathrm{pPb}$ and $\mathrm{PbPb}$ Runs at the LHC. On the other hand, diffractive nuclear parton densities (nDPDFs), determining the conditional probability to find a parton in a nucleon within the nucleus with the nucleus remaining intact (coherent diffraction), have never been measured.

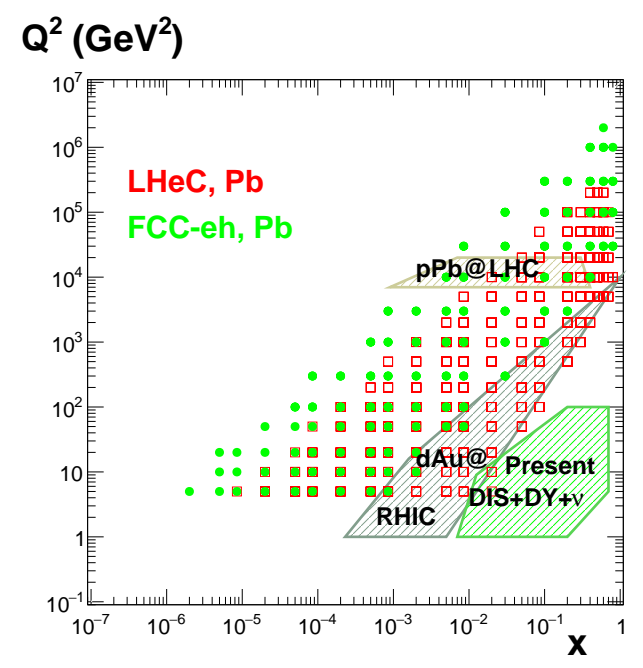

Figure 1: Kinematic region in the $x-Q^{2}$ plane covered by $e \mathrm{~Pb}$ collisions at the $\mathrm{LHeC}$ (red) and the FCC-eh (green), compared with those covered at present by fixed target DIS and DY experiments, dAu collisions at RHIC and $\mathrm{pPb}$ collisions at the LHC [3].

PDFs are ideally measured in DIS. The LHeC [7] and the Future Circular Collider in eh mode (FCC-eh) will provide electron-nucleon collisions with cms energies in the $\mathrm{TeV}$ regime and integrated luminosities $\sim 10 \mathrm{fb}^{-1}[8,9]$. It will allow a PDF fit for a single nucleus in the same setup as those for the proton, and enlarge the explored region in the $x-Q^{2}$ plane by several orders of magnitude towards small $x$ and large $Q^{2}$, see Fig. 1, thus hugely increasing our knowledge on the nuclear partonic structure and on the QCD behaviour at large energies and densities [10]. In this 
contribution I summarise the current studies on the determination of nPDFs and nDPDFs that will be fully presented in the update of the CDR in early 2019.

\section{Nuclear PDFs at the LHeC and the FCC-eh}

The $\mathrm{LHeC}$ and the FCC-eh will be able to explore a large region of phase space. Pseudodata have been generated [11] considering the main sources of uncertainties, with the kinematic cuts $\left|\eta_{\max }\right|=5$ and $0.001<y<0.95$, and conservative assumptions on the systematics. Neutral current (NC) and charged current (CC) data have been included in the EPPS16 setup previously [12] and studies are ongoing to include charm and beauty (see related a related study for the Electron-Ion Collider (EIC) in [13]). Here [14] I focus on the extraction the PDFs in Pb, using the xFitter framework [15], with the goal of estimating the ultimate achievable precision.
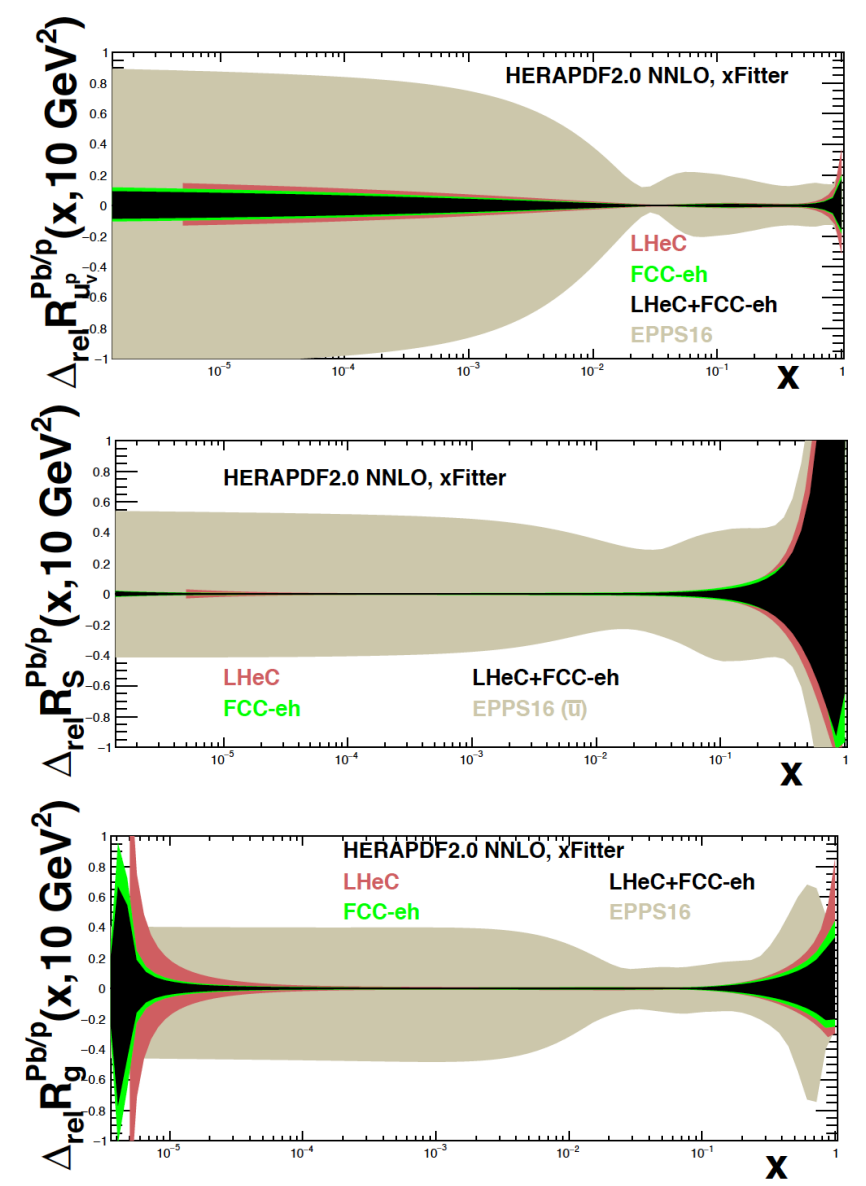

Figure 2: Relative uncertainties of the nuclear modifications factor for the valence (top), sea (middle) and glue (bottom) in an analysis of $e p$ and $e \mathrm{~Pb} \mathrm{LHeC}$ and FCC-eh NC plus CC pseudodata using xFitter (both a single set of data and all combined), compared to the results of EPPS16 [3], see the text for details.

484 (150) NC and CC data points at the LHeC (FCC-eh) have been used in the fitted region $Q^{2}>3.5 \mathrm{GeV}^{2}$, see Fig. 1. A HERAPDF2.0-type parametrisation [16] has been employed to provide both the central values for the reduced cross sections and the fit functional form; in this way, 
the uncertainty related to the functional form of the initial condition - called parametrisation bias - is absent in our study in agreement with our goal of estimating the ultimate achievable precision in the extraction of PDFs. We have worked at next-to-next-to-leading order using the RobertsThorne improved heavy quark scheme, and $\alpha_{s}\left(m_{Z}^{2}\right)=0.118$. The treatment of systematics and the tolerance $\Delta \chi^{2}=1$ are identical to that in the HERAPDF2.0 fits, as achievable in a single experiment. The results for the relative uncertainties in the nuclear modification factors for parton species $i, R_{\mathrm{i}}\left(x, Q^{2}\right)=f_{\mathrm{i} / \mathrm{A}}\left(x, Q^{2}\right) /\left[A f_{\mathrm{i} / \mathrm{p}}\left(x, Q^{2}\right)\right]$, are shown in Fig. 2. While an impressive high precision looks achievable at the LHeC and the FCC-eh, for the comparison with EPPS16 (or any other global fit) shown in the plots and with previous works in that setup [12], some caution must be taken. First, the effective EPPS16 tolerance criterium $\Delta \chi^{2}=52$ implies that naively the uncertainty bands should be compared rescaling by a factor $\sqrt{52}$. Second, the treatment of systematics is rather different, considering correlations in the xFitter exercise and taking them as fully uncorrelated (and added quadratically to the statistical ones) in the EPPS16 one. Finally, EPPS16 uses parametrisations for the nuclear modification factors for different parton species while in xFitter just the PDF combinations that enter the reduced cross sections are parametrised and employed for the fit. Work is ongoing to clarify the meaningfulness of these kind of comparisons, and to achieve full flavour decomposition using both $\mathrm{NC}$ and $\mathrm{CC}$ with heavy flavour identification.

\section{Diffractive PDFs}

As a final point, I include some comments on ongoing work [17] to establish the possibilities for constraining DPDFs in $e$ A collisions. nDPDFs have never been measured. Therefore, together with the relation between proton DPDFs and nuclear shadowing, establishing their magnitude and shape would open a new field for nuclear structure studies. In Fig. 3 we show the large enlargement of the kinematic accessible region in ep collisions compared to that studied at the Hadron-Electron Ring Accelerator (HERA), and a simulation of pseudodata in $e \mathrm{~Pb}$ using model $\mathrm{H}$ in [18]. While no model exists for a quantitative comparison of uncertainties in $e \mathrm{~Pb}$, the studies performed for $e p$ [17] promise a precise, order a few $\%$ extraction of nDPDFs.

Acknowledgments: I thank the organisers for their invitation to provide this talk, V. Guzey for providing new grids for nDPDFs and V. Radescu for help with xFitter. This work is supported by Ministerio de Ciencia e Innovación of Spain under projects FPA2014-58293-C2-1-P, FPA2017-83814-P and Unidad de Excelencia María de Maetzu MDM-2016-0692, Xunta de Galicia (Consellería de Educación) within Strategic Unit AGRUP2015/11, and FEDER. It has been performed in the framework of COST Action CA15213 THOR.

\section{References}

[1] N. Armesto, PoS DIS 2017 (2018) 107.

[2] H. Paukkunen, arXiv:1811.01976 [hep-ph].

[3] K. J. Eskola, P. Paakkinen, H. Paukkunen and C. A. Salgado, Eur. Phys. J. C 77, no. 3, 163 (2017) [arXiv:1612.05741 [hep-ph]].

[4] K. Kovarik et al., Phys. Rev. D 93 (2016) no.8, 085037 [arXiv:1509.00792 [hep-ph]].

[5] N. Armesto, PoS DIS 2018 (2018) 180. 

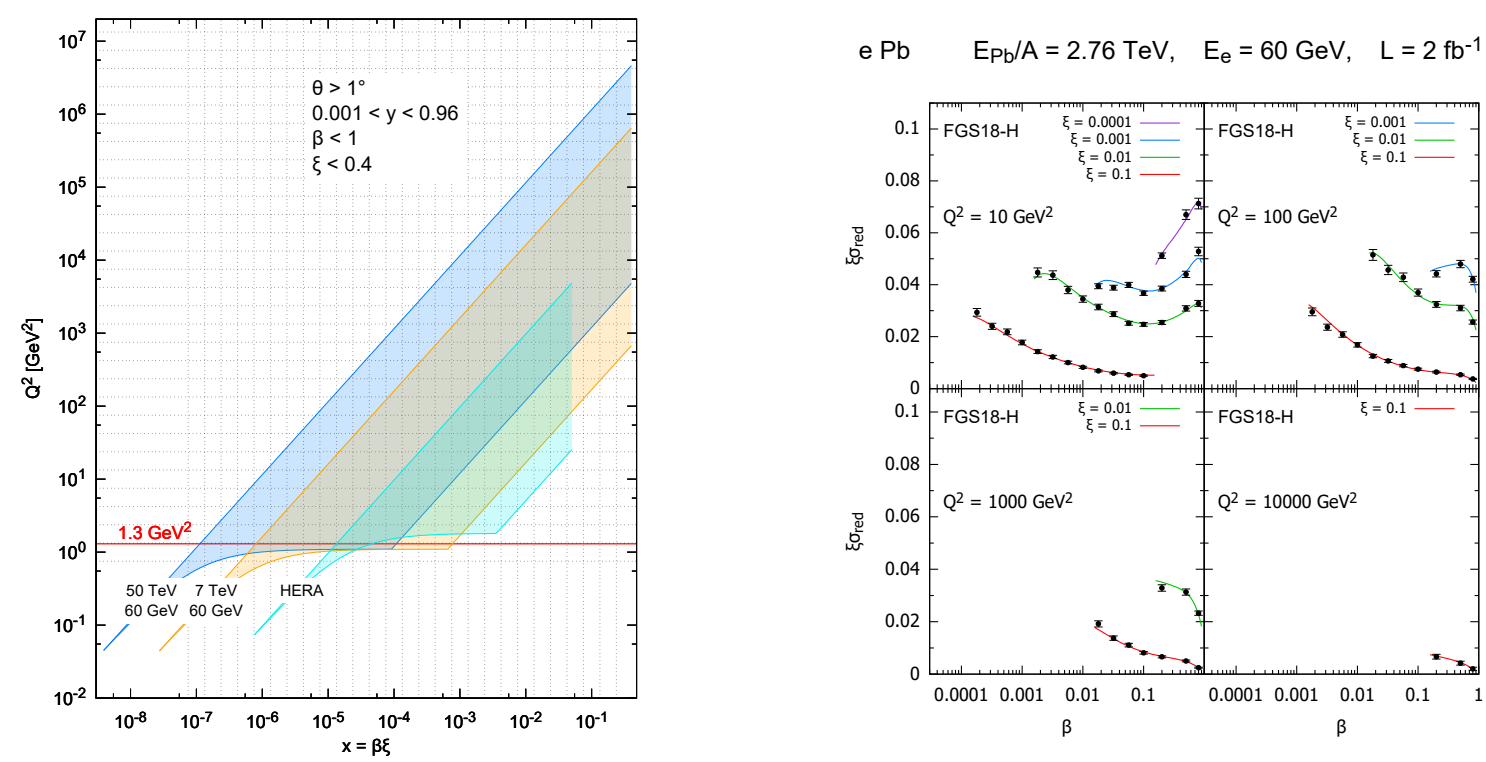

Figure 3: Left: kinematic region in the $x-Q^{2}$ plane covered by diffractive $e$ p collisions at HERA, the LHeC and the FCC-eh [17]. Right: pseudodata for diffractive reduced cross sections in $e \mathrm{~Pb}$ collisions at the $\mathrm{LHeC}$, generated using model $\mathrm{H}$ in [18].

[6] Z. Citron et al., arXiv:1812.06772 [hep-ph].

[7] J. L. Abelleira Fernandez et al. [LHeC Study Group], J. Phys. G 39 (2012) 075001 [arXiv:1206.2913 [physics.acc-ph]].

[8] F. Bordry, M. Benedikt, O. Brüning, J. Jowett, L. Rossi, D. Schulte, S. Stapnes and F. Zimmermann, arXiv:1810.13022 [physics.acc-ph].

[9] O. Brüning, J. Jowett, M. Klein, D. Pellegrini, D. Schulte and F. Zimmermann, preprint CERN-ACC-2017-0019 (2017).

[10] H. Mäntysaari, arXiv:1811.06328 [hep-ph].

[11] M. Klein, EPJ Web Conf. 112 (2016) 03002.

[12] H. Paukkunen [LHeC study Group], PoS DIS 2017 (2018) 109 [arXiv:1709.08342 [hep-ph]].

[13] E. C. Aschenauer, S. Fazio, M. A. C. Lamont, H. Paukkunen and P. Zurita, Phys. Rev. D 96 (2017) no.11, 114005 [arXiv:1708.05654 [nucl-ex]].

[14] N. Armesto, talk at the 2nd FCC Physics Workshop (CERN, January 15th-19th 2018), https://indico.cern.ch/event/618254/contributions/2833211/ attachments/1582727/2501232/armesto_FCCPW_180115.pdf.

[15] S. Alekhin et al., Eur. Phys. J. C 75 (2015) no.7, 304 [arXiv:1410.4412 [hep-ph]].

[16] H. Abramowicz et al. [H1 and ZEUS Collaborations], Eur. Phys. J. C 75 (2015) no.12, 580 [arXiv:1506.06042 [hep-ex]].

[17] N. Armesto, P. R. Newman, W. Słomiński and A. M. Staśto, to appear; A. M. Staśto, talk at the Electrons for the LHC - LHeC/FCCeh and Perle Workshop (LAL, Orsay, June 27th-29th 2018), https://indico.cern.ch/event/698368/contributions/3042049/ attachments/1676701/2692163/stasto_jun28.pdf.

[18] L. Frankfurt, V. Guzey and M. Strikman, Phys. Rept. 512 (2012) 255 [arXiv:1106.2091 [hep-ph]]. 